\title{
The new General Medical Services (GMS) contract and the National Strategy for Sexual Health and HIV in England
}

\section{Background}

The National Strategy for Sexual Health and HIV 1 recommends the development of holistic, integrated, sexual health services with three levels of service provision. The new General Medical Services (GMS) contract represents the best opportunity to date to implement the strategy across general practice.

The new GMS contract was implemented on 1 April 2004. It introduces a 33\% increase in investment to general practice over the next 3 years, and marks a shift from the old volume-based general practitioner (GP) contract to a new practice-based contract that rewards quality outcomes. It also introduces new flexibilities aimed at empowering practices to more effectively manage their own workload and improve services for patients. To date $60 \%$ of GPs in England provide primary medical services under GMS contracts and $40 \%$ under Personal Medical Services (PMS) contracts. PMS contracts are a local voluntary alternative to the nationally negotiated GMS contract. PMS was piloted from 1998 and is now a permanent contractual option. The new GMS contract introduces changes for both GMS and PMS practices. The Chair of the National Health Service (NHS) Confederation negotiating team, Mike Farrar, has described the new GMS contract as a 'landmark in the development of UK general practice'? ${ }^{2}$

The new GMS contract comprises three categories of service: essential, additional and enhanced. In addition, the contract rewards practices for the delivery of high-quality services through the Quality and Outcome Framework (QOF). Essential and additional services will be funded through the global sum allocation made to all GMS practices based on six key determinants of practice workload and circumstances, including local epidemiological, demographic, social and economic factors.

\section{Essential services}

All practices will be required to provide essential services which include the management of patients or temporary residents who are ill, terminally ill or suffering from a chronic disease. In these terms 'disease' is defined by the International Statistical Classification of Diseases and Related Health Problems (ICD-10). ${ }^{3}$ Importantly, the ICD10 includes all sexually transmitted infections (STIs) and human immunodeficiency virus (HIV) within its definitions of disease. Practices are also responsible for the provision of advice in connection with the patients' health, including relevant health promotion advice and the referral of patients to other services.

Currently there is extensive debate regarding the definition of essential services in all clinical fields, as this definition determines which services practices are expected to provide out of their global sum allocation, versus what the Primary Care Organisations (PCOs) should fund as enhanced services. In terms of sexual health, the strategy recommends that all practices should provide 'Level 1' sexual health services including sexual history taking, risk assessment, STI and HIV testing, hepatitis B immunisations, pregnancy testing and referral for termination of pregnancy. These services would have to be agreed with practices as essential primary medical services, and PCOs should be supporting the training and resource needs of practices aiming to provide these Level 1 services.

\section{Additional services}

There are seven additional services, and these include cervical cytology and contraceptive services. All practices are expected to provide additional services and have a preferential right to do so, although they do have an ability to opt out of provision in accordance with fixed UK-wide rules. The opt-out clause has been introduced in order to allow practices to more effectively manage their workload within the capacity and capability of the practice team. Practices can also opt out of additional services if they have historically not provided the service or they have a conscientious objection to provision of the service. Where a practice opts out of providing an additional service, the responsibility for provision and a percentage of the global sum allocation will revert to the PCO. For example, if a practice opts out of cervical screening, all responsibility will revert to the alternative provider of the service for that practice's registered population. The opt-out price for contraceptive and cervical cytology services is $2.4 \%$ and $1.1 \%$ of the global sum allocation, respectively, which in 2004-2005 will be approximately $£ 2658$ and $£ 1203$ per average GP with a list of around 1800 patients with average population needs and service delivery costs. Anecdotal evidence indicates that practices that previously provided contraceptive or cervical cytology services are not opting out under the new GMS contract.

Under the old GMS contract the funding for contraceptive services included intrauterine devices (IUDs) and implants, but due to the new funding regime these services are explicitly excluded under the new GMS contraceptive service definition. Sterilisation and condoms were not included under the old GMS contraceptive funding and therefore are implicitly excluded from additional contraceptive services. The contractual regulations for contraceptive services have been improved and expanded under the new contract. Therefore, in addition to providing advice, medical examination, treatment and the supply of contraceptives, the regulations now include a contractual obligation to provide information about the full range of contraceptive methods, initial advice about sexual health promotion and STIs, and clear referral to specialist sexual health services where necessary. If these new regulations are performancemanaged effectively, they have the potential to improve the quality and range of contraceptive services available to patients.

\section{Quality and outcome framework}

The new GMS contract is underpinned by the introduction of the QOF. The QOF is a points-based system that rewards practices for the depth and breadth of quality across clinical, organisational, additional services and patient experience domains. There are 1050 points in the QOF, with each point worth approximately $£ 75$ per practice in Year 1 , increasing to $£ 120$ per practice in Year 2 . There are 22 quality points available for cervical cytology services, and two points for contraceptive services. The contraceptive points relate to a written policy for responding to requests for emergency contraception, and a policy for providing preconceptual advice. The contraceptive services indicators require particular tasks to be performed in relation to a target population, and the 
points available in relation to these indicators will only be obtainable, and then in full, if the task is accomplished.

\section{Enhanced services}

The third service category is that of enhanced services. Enhanced services are essential or additional services delivered to a higher specified standard, or services not provided through essential or additional services. Enhanced services are funded from the PCO unified budget, which has been increased as part of the new GMS agreement. There is a guaranteed minimum level of investment that each PCO must spend on enhanced services. There are three types of enhanced services as follows:

1. Directed Enhanced Services (DES): there are six DESs under national direction with national specifications and benchmark pricing which all PCOs must commission to cover their relevant populations.

2. Nationally Enhanced Services (NES): there are 12 NESs with national minimum specifications and benchmark pricing, but not mandatory for PCOs to commission.

3. Locally Enhanced Services (LES): these provide PCOs with opportunities to commission locally necessary services to locally agreed specifications and pricing.

There are two enhanced services for sexual health services, namely the IUD NES and the More Specialised Sexual Health Services NES. The IUD NES includes the provision of the intrauterine system, and recommends a national specification for high-quality delivery and a fixed per item price. The More Specialised Sexual Health Services NES allows for the commissioning of STI and HIV services over and above essential and additional services. Either of these NESs can be altered to meet local circumstances and commissioned as LESs where necessary.

\section{Patient Services Guarantee}

Whilst the commissioning of NESs is not compulsory, PCOs do have a responsibility to meet the Patient Services Guarantee (PSG), which ensures that patients will continue to be offered at least the range of services that they enjoyed under the old GMS contract. The services may be made available to the patient from somewhere other than their own local practice and provided by the most suitable provider in order to ensure patient choice and minimise any travel times. Both IUDs and contraceptive implants were provided under the old contract and therefore are guaranteed under the PSG. The Faculty of Family Planning and Reproductive Health Care, the Royal College of Nursing, fpa (Family Planning Association) and Royal College of General Practitioners Sex Drugs and HIV Task Group have published a recommended locally enhanced service specification for contraceptive implants (available at http://www.ffprhc.org.uk).

\section{Personal medical services}

PMS practices will retain their existing PMS budget and therefore will not be allocated a global sum. They will have to provide an equivalent range of essential services, and will have the same ability to opt out of additional services with a nationally agreed opt-out price. PMS practices will have equivalent access to the national QOF, although some quality indicators were originally calculated into PMS contracts and therefore 174 points will be deducted from PMS QOF achievements. PMS practices will have the additional flexibility of being able to alter elements of the QOF against national guidelines. For example, a PMS practice providing services to a university student population may exchange clinical points that are less relevant to their population, such as coronary heart disease, for sexual health clinical quality points where they are providing sexual health services. In this way PMS practices will have the opportunity to negotiate local QOFs with their PCO in order to most effectively represent the services they provide to their registered population. PMS practices will also have equivalent opportunities in relation to enhanced services.

\section{Summary}

In summary, the new GMS contract provides a number of opportunities to further implement the National Strategy for Sexual Health and HIV, and a mechanism through which PCOs could secure the provision of Level 1 sexual health services in all general practices. This presents the best opportunity to date to redesign sexual health services around the needs of the patient and improve the range and quality of sexual health services available in general practice. This will require multidisciplinary working, strong commitment from PCOs to support practices in delivering at least all Level 1 services, and a prioritisation of resources for Level 2 and 3 sexual health services, as recommended in the commissioning toolkit. ${ }^{4}$

Statements on funding and competing interests

Funding. None identified.

Competing interests. None identified.

Mitzy Gafos, MSc

Primary Care Lead, Sexual Health Team, Department of Health, Skipton House, Room 580D, 80 London Road, London SE1 6LW, UK. Tel: +44 (0) 2079721527

References

1 Department of Health. National Strategy for Sexual Health and HIV. London, UK: Department of Health, 2001.

2 New General Medical Services (GMS) Contract. British Medical Association (BMA) and National Health Service (NHS) Confederation, 2003. http://www.dh.gov.uk.

3 World Health Organization (WHO). International Statistical Classification of Diseases and Related Health Problems (ICD-10). Geneva, Switzerland: WHO, 1992

4 Department of Health. Effective Commissioning of Sexual Health and HIV Services: A Sexual Health and HIV Commissioning Toolkit for Primary Care Trusts and Local Authorities. London, UK: Department of Health, 2003. therefore all enquiries should be directed to the new Primary Care lead: Kate.Henderson-Nichol@doh.gsi.gov.uk. Mitzy Gafos is now working in South Africa but can be contacted at mgafos@africacentre.ac.za. 